\title{
A new convective cloud field model based on principles of self-organisation
}

\author{
F. J. Nober ${ }^{1}$ and H. F. Graf ${ }^{1, *}$ \\ ${ }^{1}$ Centre for Atmospheric Science, University of Cambridge, UK \\ *also at: Max-Planck-Institute for Meteorology, Hamburg, Germany
}

Received: 21 January 2004 - Published in Atmos. Chem. Phys. Discuss.: 1 July 2004

Revised: 18 August 2005 - Accepted: 27 September 2005 - Published: 20 October 2005

\begin{abstract}
A new cumulus convection parameterisation is presented in this paper. The parameterisation uses an explicit spectral approach and determines, unlike other convection schemes, for each convection event a new cloud distribution function regarding to the given vertical temperature and humidity profiles. This is done by using a one dimensional cloud model to create a spectrum of different clouds. The interaction between all non convective physical processes in the AGCM and all different clouds is taken into account to calculate a selfconsistent cloud spectrum. The model has been implemented in the ECHAM5 AGCM and tested against a large eddy simulation model. The representation of a shallow cumulus cloud field by the AGCM could be much improved. Diurnal cycle, cloud cover, liquid water path and the vertical structure of the mass flux, determined by the new convection scheme are close to the large eddy simulation, whereas the standard convection scheme failed in simulating this convection episode.
\end{abstract}

\section{Introduction}

Cumulus convection is one of the major problems in global climate modelling (Emanuel, 1994). A reasonable treatment of the physical processes associated with convective clouds is of great importance for many other physical processes in an Atmospheric General Circulation Model (AGCM). Convection to a large degree controls the vertical transport of moisture, chemical tracers, energy and momentum. When precipitation forms in convective clouds, the net latent heat release (due to condensation of water vapour to cloud droplets and afterwards precipitating raindrops) leads to a supply of available potential energy in the free atmosphere. This couples convection to the large scale dynamics. Cumulus con-

Correspondence to: H. F. Graf

(hfg21@cam.ac.uk) vection not only directly takes part in the global energy and water cycle (transport), but also indirectly by the outflow of cloud water at the top of convective clouds. Within an AGCM cloud processes are separated into convective and stratiform clouds. Outflowing water from convective clouds is used as a source for stratiform clouds. Therefore, convection not only leads to a redistribution of moisture and energy within cumulus towers themselves, but also to a decoupling of these quantities from their primary sources. Stratiform clouds in turn have great importance for the radiation budget of the Earth. The problem of cumulus parameterisation is still an unsolved problem in climate modelling (Emanuel and Raymond, 1993; IPCC, 2001). Most of the current cumulus convection parameterisations are formulated as mass flux schemes (determine the overall mass flux of all cumulus clouds in one AGCM grid column) (Anthes, 1977; Emanuel, 1994; Emanuel and Raymond, 1993; Tiedtke, 1989). Several different attempts have been made to parameterise convection. These approaches are different in complexity and the underlying physical assumptions. While Kuo (1965, 1974) assumed a statistical equilibrium for water substance, in the convective adjustment approach (Betts, 1986; Betts and Miller, 1986) an unstable temperature profile is adjusted back towards a profile that is neutral or nearly neutral to convection. Other convection schemes are based on cloud models (Tiedtke, 1989; Arakawa and Schubert, 1974; Kreitzberg and Perkey, 1976, 1977; Kain and Fritsch, 1990). The model presented in this paper belongs to this group. Beginning with Arakawa and Schubert (1974) the idea of an explicit cloud spectrum was introduced in the field of convection parameterisation. This scheme describes a spectrum of mass fluxes. The mass flux approach causes a lack of information about cloud dynamics and microphysics. In contrast to Arakawa and Schubert (1974), Donner (1993), Donner et al. (2001) and Naveau and Moncrieff (2001) describe a spectrum of simplified clouds and not mass fluxes. Cloud dynamical and microphysical structures are represented in a more precise 
way, but both schemes are based on either observations or high resolution simulations.

The presented Convective Cloud Field Model (hereafter CCFM) is not based on any observations, but on a theoretical concept. It determines for each AGCM grid column an explicit spectrum of different clouds. Therefore, the information about the actual cumulus convection state in a grid column is not restricted to an averaged mass flux, but includes the number of different clouds which, in principle, are able to develop under the given atmospheric conditions. The different clouds, which are calculated by a simple entraining parcel model, are defined by their different initial radii and initial vertical velocity. The degree to which each cloud type participates in the whole cloud field is determined by the cloud field model with respect to the given vertical profiles of temperature and humidity in the grid column. The choice of the cloud model to define the different clouds is very flexible. Very simple cloud models are possible, but also more complex ones that describe more realistic clouds (including dynamic and micro-physical information) than simple mass flux approaches do. To determine the cloud field, the CCFM takes into account the effect of all non-convective processes calculated by the AGCM and (which makes the procedure self consistent) the cloud-cloud interaction between all cloud types. The final calculation of the cloud field follows an approach known from population dynamics that was developed to describe the interaction between different biological species (Volterra, 1931; Lotka, 1925). Therefore, in contrast to other recent spectral convection parameterisations, the cloud distribution function in our model is not extracted from a specific convection episode. This does not mean that the approach necessarily leeds to better results. However, once having shown that the new approach works in principle, there are many possibilities for further improvement. Our paper is structured as follows: In Sect. 2 we give a detailed description of our approach. This is followed by a comparison of the CCFM with the standard ECHAM5 and a Large Eddy Simulation (LES) model in Sect. 3. After discussing the performance of the model (with respect to other attempts to overcome the shortcomings of traditional mass flux schemes) in Sect. 4, we end up with final conclusions.

\section{Model description}

The physical problem we want to describe is to a large degree a self-organised system. Considering an atmospheric column, as we have to describe it in an AGCM, the situation is as follows: There is a forcing term, which is actually given by every process that can create instability, and cloudcloud interaction. When convection starts, normally not only one cloud forms. Different clouds develop, modify their surroundings - reduce instability - and affect each other. The resultant cloud field is due to self-organisation of different clouds. Other external control mechanisms (e.g. wind shear) also play an important role in the organisation of convective cloud fields. As a first order approximation we will neglect those effects in this paper. Since mass flux schemes describe the integrated effect of a whole cloud ensemble, the effect of a cloud ensemble, including the cloud - cloud interactions, has to be described by empirical parameters. The use of empirical parameters leads to very efficient numerical codes. Therefore, mass flux schemes can be used for long time simulations of global climate. On the other hand, these parameters are not able to represent the complete phase space of possible cloud configurations. Convection parameterisation schemes based on mass flux approach in general are tuned to simulate a few convective cases in a reasonable way. Using such parameterisations in a global model implies that it works reasonably in all meteorological situations that appear in the model climate (Siebesma and Holstlag, 1996). The main purpose of our work is to treat the problem of cumulus parameterisation from the view of self-organisation.

The proposed model follows the idea of quasi-equilibrium that was first formulated by Arakawa and Schubert (1974). This idea to a large degree defines how the convective scheme has to be inserted into the hosting AGCM. Since we tested the model in the ECHAM-AGCM (Roeckner et al., 1996) we confine our explanation to this model. There are no principal differences to other AGCMs.

A significant feature of the presented CCFM is its modular structure. This makes the model very flexible and allows changes of the separate components. In principle, we have three main parts in the model: The first part is a cloud model. Here we use, as a first step, a simple entraining parcel model. This is used to define the cloud types which are able to develop under a given meteorological situation. The next step is to define the cloud forcing and the cloud-cloud-interaction coefficients for these specific clouds and the specific meteorological situation. The last step is to use the Lotka-Volterra equation (Volterra, 1931; Lotka, 1925) from population dynamics to calculate the development and the final state of the cloud spectrum. This equation represents the closure in our approach. Each of these three parts should (and will) be object of our further investigations. We want to underline here that so far no specific tuning was applied to the CCFM or any of its parts. The flow diagram is shown in Fig. 1 and the three parts will be described in the following section.

\subsection{One-dimensional cloud model}

The one-dimensional cloud model is based on a computer model developed in the late 1960's at the Pennsylvania State University (Weinstein and MacCready, 1969). We used this model because of its computational efficiency. Nevertheless, it resolves more dynamical and microphysical features than common mass flux schemes do. It was tested for the maximum top height of convective clouds by Graf and Teubner (1988) against three years worth of RADAR observations, who showed that observed maximum cloud top heights and 
modelled cloud top heights are highly correlated $(r=0.8)$ for a large number of different cases. The model is a steady state Lagrangian solution of a set of equations for temperature, cloud radius, vertical velocity, cloud water/ice and rain water/ice. The thermodynamic and dynamic calculations are numerical analogies of the classical parcel method with entrainment. The entrainment rate $\left(=0.2 / r_{\text {updraft }}\right)$ is the same as in the original setup (Weinstein and MacCready, 1969; Graf and Teubner, 1988) and has not been adjusted for our case. The updraft radius ( $r_{\text {updraft }}$ ) changes due to vertical acceleration, entrainment and density effect. The equations are based on the first law of thermodynamics and the vertical equation of motion. Cloud microphysical calculations follow the well known parameterisation developed by Kessler (1969) and include ice formation (Ogura and Takahashi, 1971) . To run the model requires a set of vertical profiles (temperature, humidity) and initial values for cloud base radius ( $\left.r_{\text {initial }}\right)$ and cloud base vertical velocity ( $\left.w_{\text {initial }}\right)$. The indefiniteness of these two parameters opens the possibility for creating a cloud spectrum. As mentioned before, the first step is to define, for a given grid column and at a given time step, a spectrum of different clouds which in principle are able to develop under this meteorological situation. The physical parameterisations in ECHAM follow a certain sequence: Radiation, vertical diffusion, gravity waves, convection, stratiform clouds. We assume the following idealised but not too artificial situation: Let the vertical profile in a specific grid column be a neutral one. Actually it could be any profile except an unstable one. Then the physical processes (namely radiation and advection), that are specified before convection, are able to create convective instability. At the time convection is activated there are probably two sets of vertical profiles available: A neutral one from the last time step (which we call $T M$ for temperature and $H M$ for humidity) and an unstable one which is just the sum of $T M$ and $H M$ and the tendencies of the current time step. We call them $T$ and $H$. So we have a stable situation and an unstable situation. The vertical distribution of convective instability determines the cloud spectrum. We take the "unstable" set of profiles (temperature and Humidity, provided by ECHAM5), since this set is susceptible for convection, and run the one - dimensional model many times with different initial values for cloud base radius and cloud base upward velocity. Practically, this is done by choosing for both quantities an upper and a lower boundary and an increment. Let us assume that we have $K$ initial radii $\left(r_{\min }\right.$, $\left.r_{\min }+\Delta r, \ldots, r_{\min }+\Delta r(K-1)\right)$ and $L$ initial vertical velocities $\left(w_{\min }, w_{\min }+\Delta w, \ldots, w_{\min }+\Delta w(L-1)\right)$. We end up with $K \cdot L$ different couples of initial conditions that lead to $K \cdot L$ different cloud types. Each cloud type is completely defined by the output-profiles of the one-dimensional model. The difference in these cloud types depends on the increment of $r$ and $w$ and on the given meteorological situation. Therefore, cloud type number $i=1, \ldots, K \cdot L$ is defined by the initial radius, the initial vertical velocity, environmental tempera-

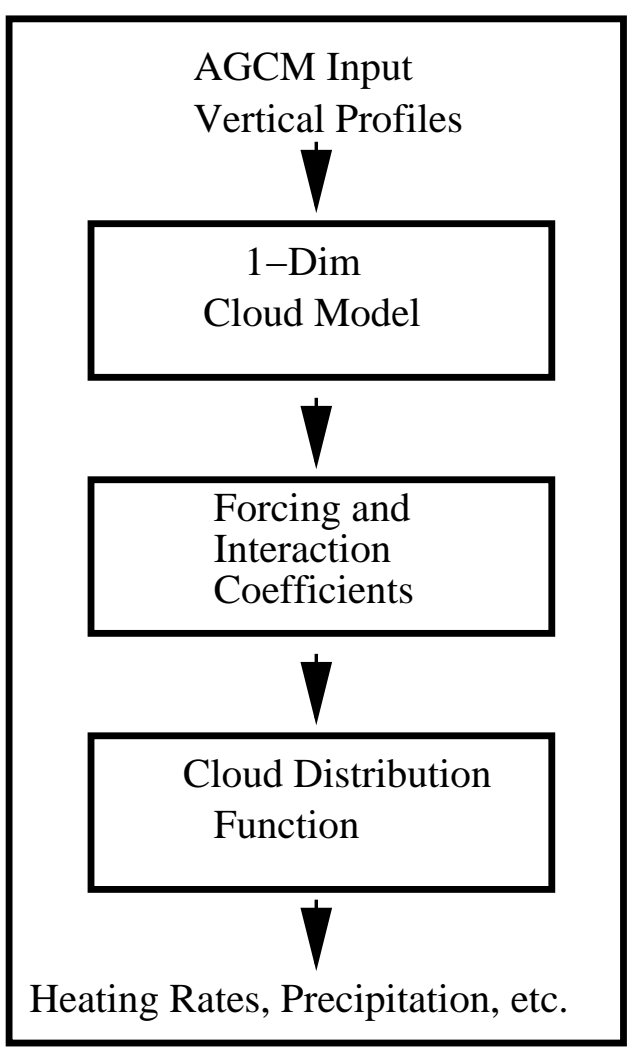

Fig. 1. Flow diagram of the CCFM.

ture and humidity profiles, and the cloud model.

In Fig. 2-3 we show different cases of cloud vertical velocity profiles as examples for the variety of resulting cloud types arising from different meteorological conditions. The shown figures (Fig. 2-3) are characterised by convective instability. The difference between the two cases are the vertical profiles of temperature and humidity. The temperature profile is dominating and is therefore shown in the Figures. In Fig. 2 and 3 we find two cases of convection. While in the first case convection is weak and shallow (max. vertical velocity $\approx 7 \mathrm{~m} / \mathrm{s}$, cloud depth $\approx 2500 \mathrm{~m}$ ), in the second case, according to the temperature gradient, the upward motion appears to be much stronger. In this case we have three distinct levels of cloud tops ( $\approx 1000 \mathrm{~m}, \approx 7000 \mathrm{~m}, \approx 10000 \mathrm{~m}$ ). This is caused by a small change of the temperature gradient at about $1000 \mathrm{~m}$ and an inversion at about $6500 \mathrm{~m}$. Clouds with small initial radii can only reach the first level $(\approx 1000 \mathrm{~m})$. Those with larger initial radii can reach the next level and a few clouds with the largest radii can even overshoot the inversion at $6500 \mathrm{~m}$.

The results with varying initial radii show that even relatively small changes in the vertical temperature gradient, namely the strength and heights of inversions, may excert very strong effects on the spectrum of convective clouds that may potentially develop in the specific environment. In these 

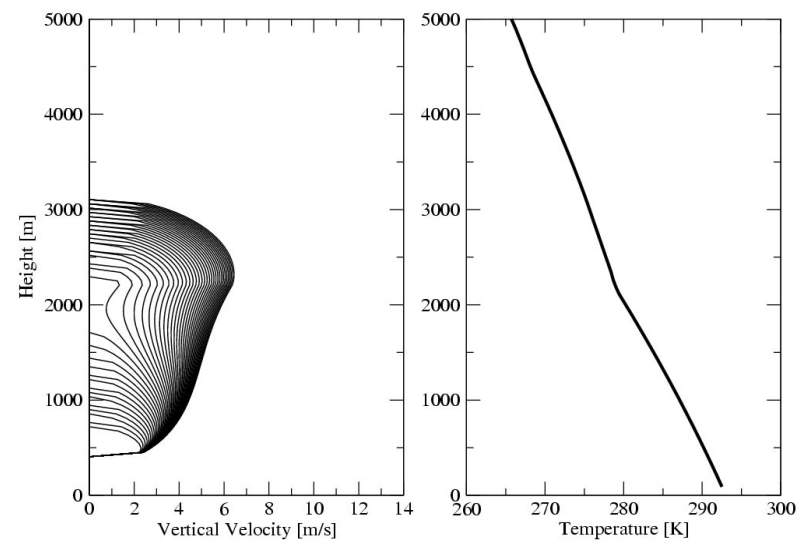

Fig. 2. Cloud updraft velocity and temperature profile; Different colours indicate different initial cloud base radii.
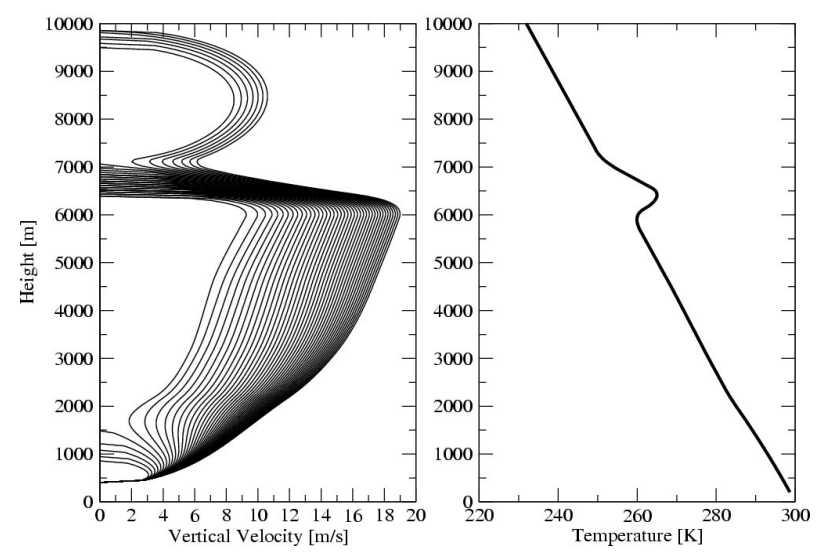

Fig. 3. Cloud updraft velocity and temperature profile for different initial cloud base radii.

calculations the interaction between the single clouds is not taken into account. This process will further change the cloud spectrum. A subset of the manifold of possible clouds will finally form the spectrum of convective clouds. This will be treated in the following section.

\subsection{Forcing and interaction coefficients}

To formulate our model concept, we make extensive use of the CAPE (Convective Available Potential Energy) formalism. The model as proposed is constructed such that the resulting cloud ensemble consumes as much CAPE as possible. Recent observations indicate that this might be wrong. Regarding Zhang (2002) convection does not completely consume instability generated by other processes. This fact can be included in the present model by adjusting the forcing coefficients $F_{i}$. It would correspond to an "effective" forcing. Such tuning, however, was not applied here since it requires a large amount of testing and comparison with observations, which will be done in the future but is beyond the scope of the current paper.

\subsubsection{Cloud forcing}

First we need information which cloud types are actually forced or supported by external processes. As mentioned before, we have available two sets of vertical profiles when running the convection scheme. We called them $(T M, H M)$ for the "neutral" from the last time step and $(T, H)$ for the "potentially unstable" one. Since we have run the cloud model in the unstable environment $(T, H)$, we have a third set of profiles (separately for each cloud type) available which is the in-cloud-temperature $T_{c_{i}}$ of cloud type number $i$. We now define the "cloud available potential energy content" by:

$\mathrm{CAPE}_{i}(T)=g \int_{\text {Base }_{\mathrm{i}}}^{\mathrm{LNB}_{\mathrm{i}}} \frac{T_{c_{i}}-T}{T} d z$

and

$\mathrm{CAPE}_{i}(T M)=g \int_{\text {Base }_{\mathrm{i}}}^{\mathrm{LNB}_{\mathrm{i}}} \frac{T_{c_{i}}-T M}{T M} d z$

where $T=T M+T_{\text {tend }} \Delta t$, Base $\mathrm{i}_{\mathrm{i}}$ is the base of cloud type $i$ and $\mathrm{LNB}_{\mathrm{i}}$ the Level of Neutral Buoyancy of cloud type $i$. The relative change of the "cloud potential energy content" due to all non-convective processes during the current time step can therefore be expressed as

$F_{i}=\frac{\operatorname{CAPE}_{i}(T)-\mathrm{CAPE}_{i}(T M)}{\mathrm{CAPE}_{i}(T) \Delta t}$

The denominator in this expression $\left(\operatorname{CAPE}_{i}(T)\right)$ can be assumed to be always positive. In case it is zero the respective cloud per definition does not exist. It may be helpful to note that $F_{i} \times \operatorname{CAPE}_{i}(T)$ can be understood as the time derivative of $\mathrm{CAPE}_{i}(T M)$ with respect to the effect of all non-convective processes.

$\mathrm{CAPE}_{i}(T) F_{i}$

$$
\begin{aligned}
& =\frac{\operatorname{CAPE}_{i}(T)-\mathrm{CAPE}_{i}(T M)}{\Delta t} \\
& =\frac{\mathrm{CAPE}_{i}\left(T M+T_{\text {tend }} \Delta t\right)-\mathrm{CAPE}_{i}(T M)}{\Delta t}
\end{aligned}
$$

with

$\frac{d \mathrm{CAPE}_{i}(T M)}{d t}$

$=\lim _{\Delta t \rightarrow 0}\left\{\frac{\mathrm{CAPE}_{i}\left(T M+T_{\text {tend }} \Delta t\right)-\mathrm{CAPE}_{i}(T M)}{\Delta t}\right\}$ 


\subsubsection{Cloud-cloud interaction}

The cloud-cloud interaction is treated similar to the cloud forcing by external processes. First we calculate the effect of each cloud type on the environment $(T, H)$. We call the resulting profiles $\left(T_{j}, H_{j}\right)$, where $j$ is the index that indicates the cloud types. As in the former section, we are interested in the effect of the change in the vertical profiles on the cloud potential energy. According to Eq. (1) we define:

$\operatorname{CAPE}_{i}\left(T_{j}\right)=g \int_{\text {Base }_{i}}^{L N B_{i}} \frac{T_{c_{i}}-T_{j}}{T_{j}} d z$

where $T_{j}=T+T_{\text {tend }_{\mathrm{j}}} \Delta t$ and $T_{\text {tend }_{\mathrm{j}}}$ is the tendency due to the effect of cloud type $j$ on the temperature profile $T$. The relative change of cloud potential energy of cloud type $i$ due to the effect of cloud type $j$ is therefore:

$K_{i j}={\frac{\operatorname{CAPE}_{i}\left(T_{j}\right)-\mathrm{CAPE}_{i}(T)}{\mathrm{CAPE}}}_{i}(T) \Delta t$

$K_{i j} \operatorname{CAPE}_{i}(T)$ is again the time derivative of $\operatorname{CAPE}_{i}(T)$ with respect to the effect of cloud $j$.

$$
\begin{aligned}
& \frac{d \operatorname{CAPE}_{i}\left(T_{\text {effect }(\mathrm{j})}\right)}{d t} \\
& =\lim _{\Delta t \rightarrow 0} \frac{\operatorname{CAPE}_{i}\left(T+T_{\text {tend }_{\mathrm{j}}} \Delta t\right)-\mathrm{CAPE}_{i}(T)}{\Delta t}
\end{aligned}
$$

\subsection{Lotka-Volterra equation}

Now we are prepared to formulate our master-equation to calculate the resulting cloud spectrum. The Lotka-Volterra equation which is used in population dynamics (Haken, 1977,1983 ) is given by:

$\frac{d n_{i}}{d t}=n_{i} F_{i}+\sum_{j=1}^{N} K_{i j} n_{i} n_{j}$

This equation was introduced by Volterra as a model for the competition of $N$ different biological species (Lotka, 1925; Volterra, 1931; Murray, 1993). $F_{i}$ is a parameter depending on the environment and on the species number $i$. It describes the support of species number $i$ by external processes (e.g. food supply). $\mathcal{K}_{i, j \in 1, \ldots, N}$ is the interaction matrix. The coefficient $K_{i j}$ describes the interaction between species $i$ and $j$. Or more precisely, since the matrix is not necessarily diagonal, it describes the effect of species $j$ on species $i$. Depending on the signs of the interaction coefficients the system is called a cooperative $\left(K_{i j} \geq 0\right)$, a competitive $\left(K_{i j} \leq 0\right)$, or a prey - predator one $\left(K_{i j} K_{j i} \leq 0\right)$. The various systems have been extensively analysed. They have proven their ability to describe at least some features of a variety of complex systems.

The analogy that we proclaim is to treat different cloud types as different species. Biological species as described by the Lotka-Volterra equation are in competition $\left(K_{i j} \leq 0\right.$, $\left.F_{i} \geq 0\right)$ for an external food supply rate. In such a simple model the supply of food is the reason for a species to survive or to grow. The analogy to convective clouds is straightforward. The reason for convective clouds to form is convective instability ("food supply" for convective clouds). Additionally, each cloud type acts on its environment in a well defined way and tends to reduce instability. Therefore each cloud tends to reduce somehow the "food-supply" for all other cloud types $\left(K_{i j}\right)$ including itself $\left(K_{i i}\right)$.

In Eq. (9) $n_{i}$ is the number of clouds of type number $i$ that appear in the given grid column under the given meteorological situation (temperature and humidity profiles).

Term $\left(F_{i} n_{i}\right)$ represents the external forcing on the cloud field. The relative ratio of the different components $\left(F_{i}, i=1, \ldots, N\right)$ shows which cloud types are favoured by the non-convective forcing. The $n_{i}$ in this part reflects that this forcing is assumed to be proportional to the actual amount of each cloud type. Perhaps the more interesting term is $\left(\sum_{j=1}^{N}\left[K_{i j} n_{i} n_{j}\right]\right) . \quad K_{i j}$ is the effect of cloud type $j$ on cloud type $i$. Analogous to the first term, this coefficient is multiplied by $n_{i}$ and additionally by $n_{j}$ since the effect of cloud type $j$ on $i$ is assumed to increase linearly with the amount of cloud type $j$. It is possible to give a first characterisation of the equation by recalling the definition of $F_{i}$ and $K_{i j}$ and discussing some physical basics: Clouds are assumed to reduce instability and to consume convective available potential energy. Therefore, $\operatorname{CAPE}_{i}\left(T_{j}\right)$ in general is smaller than $\mathrm{CAPE}_{i}(T)$ since $T_{j}$ is already somewhat stabilised due to cloud type $j$. As a consequence $K_{i j}$ in general is negative. The non-convective processes in our case are only important if they produce convective instability. Then $\mathrm{CAPE}_{i}(T)$ is larger than $\mathrm{CAPE}_{i}(T M)$ and, therefore, $F_{i}$ is positive. Thus, in the model we assume that $K_{i j}<0$ and $F_{i}>0$. If $F_{k} \leq 0$ we can ignore this special cloud type $k$, which reduces the degrees of freedom for the Lotka-Volterra equation by one dimension. Eq. (9) is finally solved numerically. In Fig. 4 we give a graphical description of the CCFM for a reduced setup with only two different cloud types to keep the picture more transparent. In the model we normally use 20-50 different cloud types.

\subsection{Vertical resolution and cloud types}

Most convection schemes work with the vertical resolution of the host AGCM (ECHAM5 standard: 19 levels from $1000 \mathrm{hPa}$ to $10 \mathrm{hPa}$ ) or with interpolated values on "halflevels". This is sufficient for mass-flux models since these are designed for such coarse resolutions. 1-dimensional cloud models, as the one used in this work, demand a finer resolution since the microphysical and dynamical 


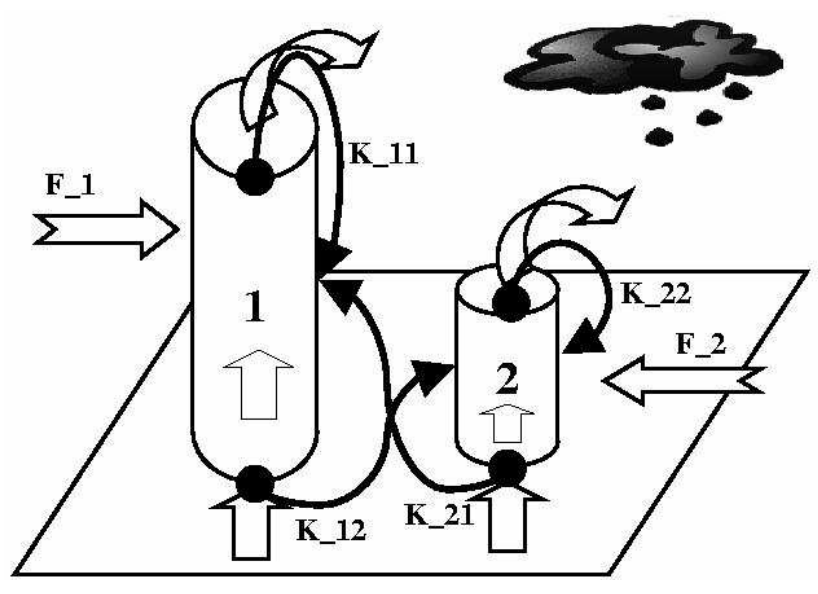

Fig. 4. Graphical description of the Convective Cloud Field Model.

calculations are more sensitive. Therefore, we interpolate the ECHAM5 standard vertical resolution to a finer vertical grid of $200 \mathrm{~m}$ vertical resolution before starting the cloud model and cloud spectrum calculation. Also the calculation of the interaction coefficients $F_{i}$ and $K_{i j}$ is performed on this finer grid and leads to more stable numerical solutions. However, we want to stress that higher vertical resolution of the host AGCM would be necessary, in order to include the small stable layers which often form in the free troposphere and which can have significant effects on the cloud spectrum especially when they occur above the freezing level.

It is obvious that the idea behind CCFM is to introduce as many different cloud types as possible. However, a limiting factor is the computational demand and the numerical accuracy of the iteration of the Lotka-Volterra equation. With increasing number of cloud types this part of the model becomes more and more problematic and time consuming. It turns out that variations in $r_{\text {initial }}$ are much more important for a large spectrum of possible clouds than variations in $w_{\text {initial }}$. Depending on the meteorological situation, there are thresholds for $w_{\text {initial }}$ and $r_{\text {initial }}$ that have to be exceeded. Otherwise no clouds will develop at all. Typical combinations for initial conditions that we used in this work are: 1-2 different $w_{\text {initial }}$ and 20-50 different $r_{\text {initial }}$. $w_{\text {initial }}$ is always chosen to be in the range of $1-2 \mathrm{~m} / \mathrm{s}$, which are typical values of vertical velocities at the base of convective clouds. $r_{\text {initial }}$ is in the range from $100 \mathrm{~m}$ up to $3000 \mathrm{~m}$. It turns out that in nearly all cases this combination leads to a spectrum of possible clouds that compares favourably with observations. $w_{\text {initial }}$ may be more important for the initial cloud droplet spectrum in case of a more complex cloud microphysics than used in this study. In the current experimental setup we use 50 different cloud types with an initial vertical velocity of $2 \mathrm{~m} / \mathrm{s}$ and initial radii between $100 \mathrm{~m}$ and $3000 \mathrm{~m}$. One task for future development of CCFM would be to couple these initial conditions to the sub-cloud layer. This, as well as the procedure to determine the cloud base (in the current version of our model by an undiluted rising air parcel until the condensation level is reached), concerns the so called "trigger function" problem. For discussion and investigation of this problem see Jakob and Siebesma (2003).

\section{Model validation}

Although the proposed CCFM actually is not a pure convection scheme but a statistical cloud field model, it can be used like a convection parameterisation within an AGCM. As input it needs vertical profiles of temperature and humidity and as output it returns information about heating rates, integrated mass fluxes, tracer transport, precipitation and moisture transport, and detrained cloud water (important as source for cloud water in stratiform clouds). We use the ECHAM5AGCM as environment for our model.

\subsection{AGCM-physics}

The dynamical core of the ECHAM model has been adopted from the European Centre for Medium Range Weather Forecasts (ECMWF) model (Roeckner et al., 1996). Vorticity, divergence, temperature, surface pressure, and mass mixing ratios of water vapour and total cloud water/ice are treated as prognostic variables. The master equations are solved on a vertical hybrid $p-\sigma$-system (19 vertical levels) using the spectral transformation method with a triangular truncation. All physical parameterisations are calculated at grid points of a Gaussian grid. For the time integration a semi-implicit leapfrog scheme is used. Physical parameterisations exist for horizontal diffusion, surface fluxes and vertical diffusion, land surface processes, gravity wave drag, and, relevant for our problem, cumulus convection, stratiform clouds and radiation.

\section{ECHAM5 standard convection:}

The ECHAM standard convection is based on the widely used bulk mass flux concept of Tiedtke (1989). The original Tiedtke scheme has been changed to include some suggestions of Nordeng (1994). These changes include organised entrainment/detrainment and the mass-flux closure (adjustment type instead of moisture convergence for deep cumulus). Cloud water that may detrain at the tops of cumulus clouds is used as a source for cloud water in stratiform clouds.

\section{Stratiform clouds in ECHAM5:}

The parameterisation of stratiform cloud cover in ECHAM5 is essentially given by a parameterisation of the horizontal sub grid-scale variability of water vapour and cloud condensate (Tompkins, 2002). This statistical cloud scheme assumes that the sub grid variability is well described by a given Probability Density Function (PDF) including two shape parameters. The cloud microphysical scheme of 
ECHAM5 uses a prognostic treatment of separate cloud ice and liquid cloud variables (Lohmann and Roeckner, 1996).

\section{Changes in the cloud-radiation interaction:}

Since all information needed by the remaining part of the ECHAM model are computed by the CCFM (especially cloud cover and cloud liquid water path as input variables for the radiation) we switched off all other cloud processes in the ECHAM model and couple our model completely to the ECHAM physics (namely radiation). This is possible because the convection episode that is simulated and presented in the next section is a definite convective case.

\subsection{Comparison to LES}

We tested the performance of the CCFM for the "ARM-case" (Atmospheric Radiation Measurement Program), which is part of the "European Project On Cloud Systems In Climate Models" (EUROCS). In the framework of this project several models of different complexity have been tested against observations. The experiment took place at the Southern Great Plains (SGP) ARM site on 21 January 1997 (ARM,EUROCS). Observations of this day show shallow cumulus clouds developed at the top of an initially clear convective boundary layer. The non precipitating cumulus cloud field showed a clear diurnal cycle starting around 8:30 h local time and disappearing around 17:30 h. This case was simulated by a number of LES-models (Large Eddy Simulation). All these models show a quite good performance to simulate both, the temporal structure (diurnal cycle) and the spatial structure (vertical mass fluxes, profiles, cloud distribution). Additionally, several global and meso-scale models were used to run this case in single column mode with prescribed surface and boundary fluxes. While LES models all show a very uniform and satisfying performance close to reality (Brown et al, 2001) the other models do not (ARM,EUROCS). Global as well as meso-scale models have serious problems to simulate a correct diurnal cycle and a reasonable cloud cover fraction and liquid water path.

Since surface and boundary fluxes are prescribed in this setup, we get rid of the problem of model inconsistency and tuning as described in the last section. Figure 5 shows the maximum cloud cover for the ARM-case from LES ${ }^{1}$, ECHAM standard, and the CCFM.

Obviously CCFM provides realistic results which differ strongly from the original ECHAM5 cloud cover scheme.

Another important cloud quantity is vertically integrated cloud water (Fig. 6). Again, CCFM produces very realistic results and the original ECHAM5 parameterisation fails to do so. Thus, two key-quantities of the shallow cumulus cloud field can be simulated by the CCFM as well as by the LES model.

\footnotetext{
${ }^{1}$ LES data and model setup kindly provided by A. Chlondt and F. Mueller.
}

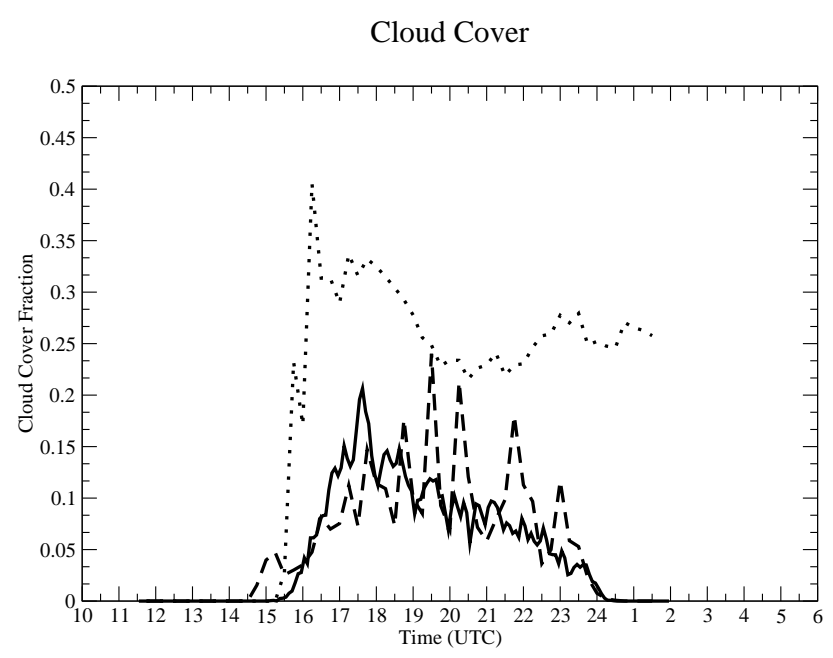

Fig. 5. Cloud cover for ARM-case; dotted line ECHAM5, solid line LES, dashed line CCFM.

Liquid Water Path

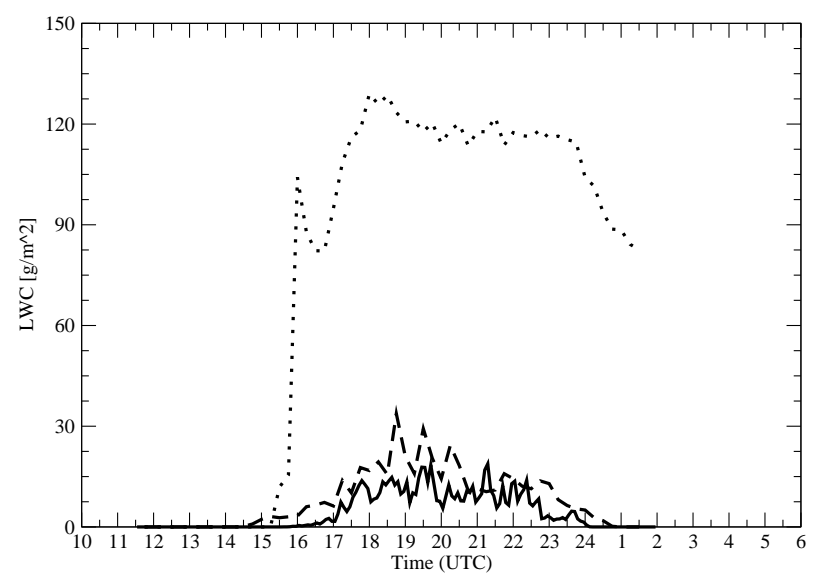

Fig. 6. Liquid water path for ARM-case; dotted line ECHAM5, solid line LES, dashed line CCFM.

Figures 7 and 8 give information about the vertical structure of the cloud field. While Figure 7 shows the vertical profiles of mass fluxes of LES, ECHAM-standard and CCFM, Fig. 8 shows the cloud distribution function calculated by CCFM.

The CCFM produces a diurnal cycle of cloudiness very close to the LES simulations. Cloud cover and liquid water path are much better simulated than in the ECHAM standard configuration. On the other hand, three main discrepancies between the CCFM and the LES model remain: Onset of convection is about one hour too early. This is probably a triggering problem of our model. A second point is the high noise particularly in the cloud cover curve of the CCFM. This behaviour is caused by the fact that there is no memory of the convective cloud state from time step to time step. Both 


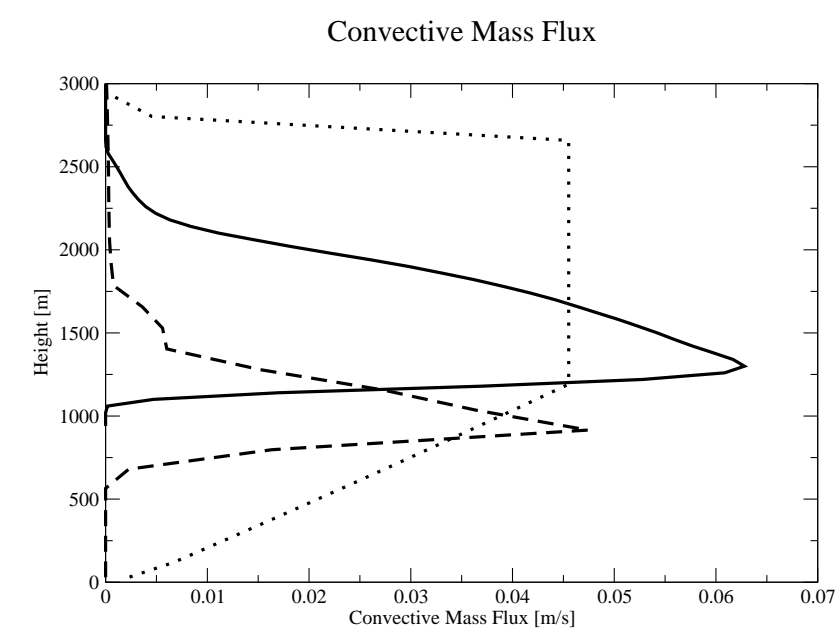

Fig. 7. Mass Flux ARM-case 20:00 UTC ; dotted line ECHAM5, solid line LES, dashed line CCFM.

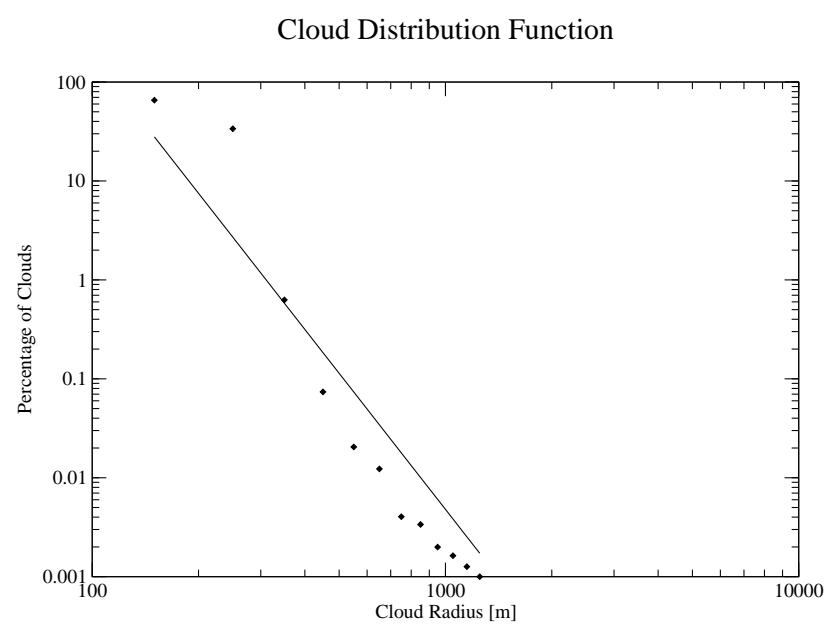

Fig. 8. Cloud distribution function from the CCFM.

problems could be solved by changing the code in ECHAM. The third and main point that has to be mentioned is the too low cloud base in CCFM (Fig. 7). The mass flux calculated by our model has a shape comparable to the LES model simulation, except the fact that it is shifted by about $500 \mathrm{~m}$ downwards. Potentially, this is caused by the interpolation from the coarse ECHAM vertical resolution to the finer resolution in the CCFM and may also be solved. Another problem may lay in the non-mixing of the initial thermal that is used to define the cloud base. Again, future work is needed to solve this problem.

The remaining discrepancies to a large degree are probably caused by the fact, that there is still no real information about the sub-grid variability of temperature and humidity profiles in our model. This shall be discussed in more detail: The slope of the power-law curve fitted to the cloud distribution function determined by our model is about -4 . This is too steep compared with observations or with the value found in LES simulations for the ARM-case by Neggers (2001). All these values are in a range of -1.5 to -2.5 . In our Cloud Distribution Function (CDF) the cloud number drops too rapidly with increasing cloud radius. A related deficiency can be identified in the mass flux curve (Fig. 7). The upper part of our mass flux curve decreases too rapidly compared with the LES data. There is a large number of small clouds but too few middle range clouds in the ensemble. The possible reason is that we have to run our model with column mean vertical profiles. This means that instability (Convective Available Potential Energy) is uniformly distributed in the whole grid area. In nature this would never be true! We would find a large fraction in the grid column with profiles which are less unstable than the grid mean profile given by the AGCM. On the other hand, we would find a small fraction in the grid column with a much more unstable vertical situation (e.g. caused by local orographic structures or just by common inhomogeneity and turbulence). Since there is no way at the time being to extract this inhomogeneity, we cannot give a solution. Possibly in the future the introduction of an inhomogeneity factor may help tuning. This problem may also arise from the fact that the interaction between the clouds is calculated by a matrix and not by a full tensor.

In spite of this bias, it is obvious that the CCFM can much better resolve the vertical structure of the convective mass flux than the standard convection scheme in ECHAM.

\section{Discussion and outlook}

The previous results showed that the Convective Cloud Field Model in principle is able to replace a well tuned common mass flux model (qualitative validation) and likewise that it simulates a shallow convection cloud field quite well (quantitative validation). Since this paper marks the beginning of our work on the model, a number of tests still has to be done. A comparison to a number of deep convection episodes (observation and high resolution simulation), a test in the global mode of an AGCM and a large number of sensitivity tests in both global mode and single column mode will be presented in upcoming papers. The CCFM was run stably in the ECHAM5 climate model over 25 years with different numbers of initial cloud types (results for January are presented in Fig. 9). Without any tuning the gross features of the global rainfall pattern are reproduced as in the original model. There are several differences as well, specifically of smaller scale nature that have to be investigated, like very high precipitation at isolated spots in the CCFM version. Important issues will be to investigate the sensitivity of the choice of the number of possible cloud types, the internal vertical resolution, and the complexity of the cloud model (e.g. microphysical parameterisations).

At this early stage we wish to discuss similarities and differences between the CCFM and the classical Arakawa 

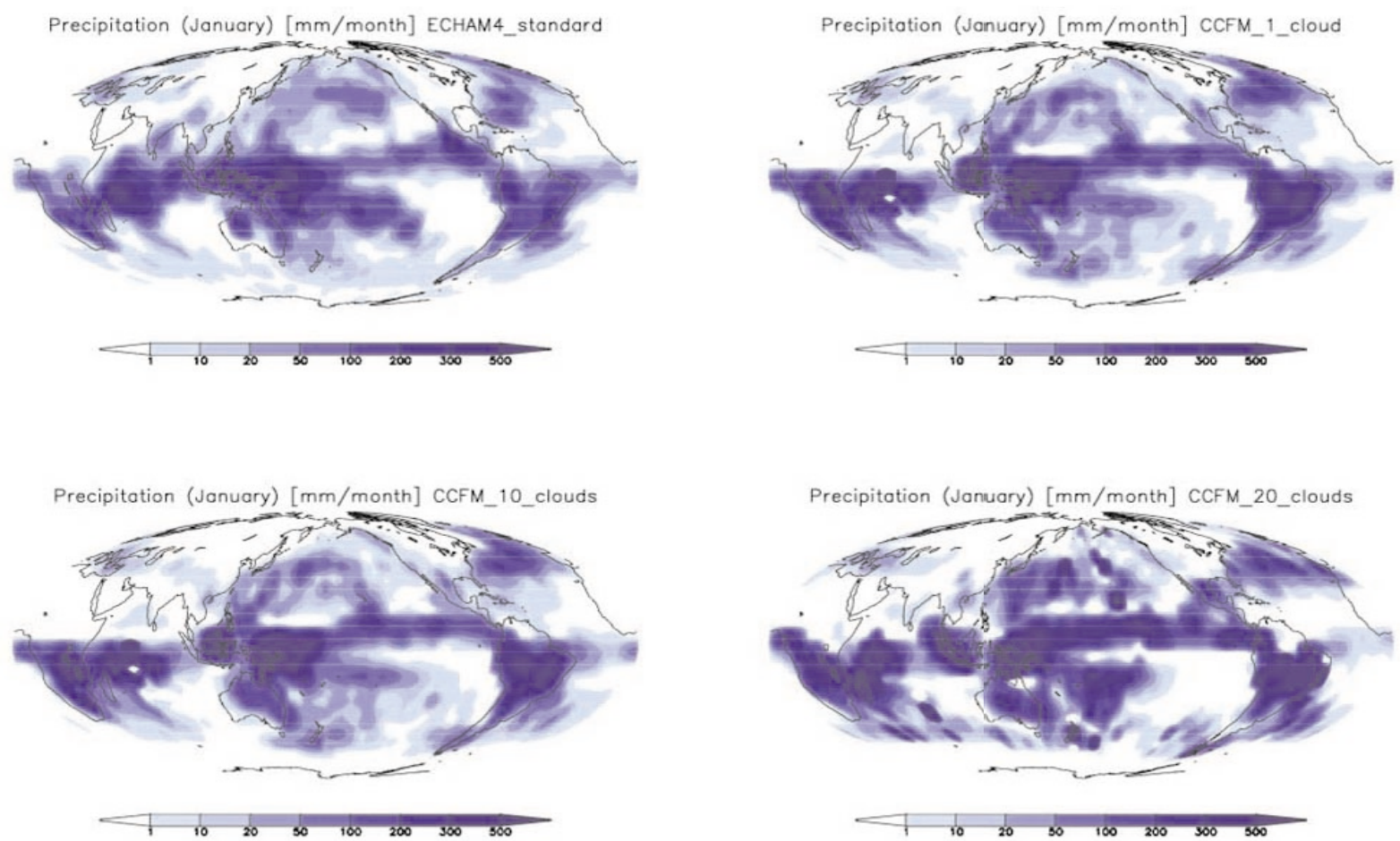

Fig. 9. Global precipitation for January [mm/month]; Upper row first picture: ECHAM standard convection, second picture CCFM with one cloud type, lower row first picture CCFM with 10 cloud types, second picture CCFM wich 20 cloud types.

Schubert approach and two other recent approaches facing the problem of convection parameterisation. The concept of CCFM is a synthesis of the classical Arakawa Schubert approach and the Lotka-Volterra approach of a competitive set of different species. The quasi-equilibrium assumption from Arakawa Schubert is extended to a quasi-“dynamic"equilibrium assumption in the CCFM. The introduction of the Lotka-Volterra equation enables the CCFM to introduce in a future version a memory for the convective state of the last time steps within an AGCM. The quasi-equilibrium would therefore be changed towards a "dynamic" or "interactive" equilibrium in which the history of convection in a given grid column influences the development of new convective clouds. This is the great potential of our approach. Already part of CCFM is the use of a cloud model instead of a mass flux model and therefore the determination of a cloud distribution function instead of a mass flux distribution function. This gives the information about convective cloud cover and convective liquid water path which is not available in mass flux schemes. Two other recent approaches were introduced by Donner (1993), Donner et al. (2001) and Naveau and Moncrieff (2001). Both follow an explicit cloud spectral strategy to describe cumulus cloud fields. While Donner (1993) and Donner et al. (2001) used direct observations of distribution functions for vertical velocities and updraft di- ameter to construct an ensemble of different clouds for a deep convection episode, Naveau and Moncrieff (2001) do so by using high resolution numerical simulations to extract a data set of vertical velocities in a cloud cluster. This data set in turn is used to determine an ensemble of clouds by applying extreme value theory. Both approaches show quite perfect results. But in both cases the cloud distribution function that characterises the cloud ensemble depends either on a specific observation or a specific simulation. The CCFM at present is possibly less successful in simulating all detailed structures of a given cloud field, but the calculation of cloud distribution functions, and therefore the resulting cloud field, depends only on the energetic situation in a given grid column and the used cloud model. Since the coefficients $F_{i}$ and $K_{i j}$ are calculated online and new for each situation, there is no need for tuning to a specific convection episode. The presented CCFM should not be seen as a parameterisation that has reached its final state of development. The number of possible cloud types in the spectrum will depend on the specific application and the program environment in which CCFM will be used. In a case with well observed temperature and humidity profiles with a high vertical resolution, the CCFM could be used as a stand alone model (without AGCM) and even more than 50 different possible cloud types. The effect of microphysics and e.g. aerosol cloud interaction can 
be taken into account and its effect on the CDF can be studied. This is not possible for a prescribed cloud spectrum that was determined by observations or high resolution numerical simulations.

\section{Conclusions}

In this paper we presented a full spectral Convective Cloud Field Model (CCFM). CCFM is based on a concept from population dynamics and therefore incorporates a method from the physics of self organising systems into the field of cloud parameterisation. The CCFM was successfully tested against a complex LES. In this case CCFM was able to simulate important quantities of a shallow cumulus cloud field very close to LES. The reference convection scheme of ECHAM5 fails to do so by a factor of 2 (cloud cover) and by a factor of 5 (liquid water path). The most important feature of CCFM is that the cloud spectrum is determined interactively. It is calculated new for each single grid column and each time step. In contrast to other spectral convection schemes, the spectrum is not fitted to a specific episode. This feature makes CCFM to also become a highly promising tool for aerosol-cloud and cloud-climate interaction. However, there will be need to investigate to what degree the concept taken from population dynamics can be applied to ensembles of cumulus clouds. Other approaches than the Lotka-Volterra equation will have to be tested and compared with high resolution models and observations.

Acknowledgements. This work was carried out within the frame work of the Smoke, Aerosols, Clouds, Rainfall, and Climate (SMOCC) project, a European contribution to the Large-Scale Biosphere-Atmosphere Experiment in Amazonia (LBA). It was financially supported by the Environmental and Climate Program of the European Commission (contract No. EVK2-CT-2001-00110 SMOCC), the Max Planck Society (MPG), the Fundação de Amparo à Pesquisa do Estado de São Paulo, and the Conselho Nacional de Desenvolvimento Científico (Instituto do Milênio LBA). We thank all members of the LBA-SMOCC and LBA-RACCI Science Teams for their support during the field campaign, especially A. C. Ribeiro, M. A. L. Moura, J. von Jouanne.

Edited by: M. G. Lawrence

\section{References}

Anthes, R. A.: A cumulus parameterisation scheme utilizing a onedimensional cloud model, Mon. Wea. Rev., 105, 270-286, 1977.

Arakawa, A. and Schubert, W. H.: Interaction of a cumulus ensemble with the large-scale environment, Part 1, J. Atmos. Sci., 31, 674-701, 1974.

ARM, EUROCS:http://www.knmi.nl/samenw/eurocs/ARM/index. html, last access: 20.10.2005.

Benner, T. C. and Curry, J. A.: Characteristics of small tropical cumulus clouds and their impact on the environment, J. Geophys. Res., 103, 28 753-28 767, 1998.
Betts, A. K.: A new convective adjustment scheme. Part I: Observational and theoretical basis, Q. J. R. Meteorol. Soc., 112, 677691, 1986.

Betts, A. K. and Miller, M. J.: A new convective adjustment scheme. Part II: Single column tests using GATE wave, BOMEX, ATEX and arctic air-mass data sets, Q. J. R. Meteorol. Soc., 112, 693-709, 1986.

Brown, A. R., Cederwall, R. T., Chlond, A., et al.: Large eddy simulations of the diurnal cycle of shallow cumulus convection over land, Q. J. R. Meteorol. Soc., 127, 1075-1093, 2001.

Cahalan, R. F. and Joseph, J. H.: Fractal statistics of cloud fields, Mon. Wea. Rev., 117, 261-272, 1989.

Donner, L. J.: A cumulus parameterisation including mass fluxes, vertical momentum dynamics, and mesoscale effects, J. Atmos. Sci., 50, 889-906, 1993.

Donner, L. J.: A cumulus parameterisation including mass fluxes, vertical momentum dynamics, and mesoscale effects: Thermodynamic and hydrological aspects in a general circulation model, J. Clim., 14, 3444-3463, 2001.

Emanuel, K. A.: Atmospheric Convection, Oxford University Press, 1994.

Emanuel, K. A. and Raymond, D. J.: The Representation of Cumulus Convection in Numerical Models, Meteorol. Monogr., American Meteorological Society, 1993.

EUROCS: http://www.cnrm.meteo.fr/gcss/EUROCS/EUROCS. html, last access: 20.10.2005.

Graf, H.-F. and Teubner, R.: Zur numerischen Bestimmung der Obergrenze konvektiver Wolken, Abh. Meteorol. Dienst DDR, 140, 83-85, 1988.

IPCC: Third assessment report, Climate change, 427-431, 2001.

Jakob, C. and Siebesma, A. P.: A new sub-cloud model for mass flux convection schemes: Influence on triggering, updraft properties and model climate, Mon. Wea. Rev., 131, 2765-2778, 2003.

Kain, J. S. and Fritsch, J. M.: A one-dimensional entraining/detraining plume model and its application in convective parameterisation, J. Atmos. Sci., 47, 2784-2802, 1990.

Kessler, E.: On the distribution and continuity of water substance in atmospheric circulation models, Meteor. Monographs, 10, Americ. Meteor. Soc., Boston, MA., 1969

Kreitzberg, C. W. and Perkey, D.: Release of potential instability. Part 1: A seqeuntial plume model within a hydrostatic primitive equation model, J. Atmos. Sci., 33, 456-475, 1976.

Kreitzberg, C. W. and Perkey, D.: Release of potential instability. Part 2: The mechanism of convective /mesoscale interaction, J. Atmos. Sci., 34, 1569-1595, 1977.

Kuo, H. L.: On formation and intensification of tropical cyclons through latent heat release by cumulus convection, J. Atmos. Sci., 22, 40-63, 1965.

Kuo, H. L.: Further studies of the parameterisation of the influence of cumulus convection on the large-scale flow, J. Atmos. Sci., 31, 1232-1240, 1974.

Lohmann, U. and Roeckner, E.: Design and performance of a new cloud microphysical scheme developed for the ECHAM generak circulation model, Clim. Dyn., 12, 557-572, 1996.

Lotka, A. J.: Elements of Physical Biology, Williams and Wilkins, Baltimore, 1925.

Mitchell, J.: Modeling cloud-climate feedbacks in predictions of human-induced climate change, Workshop on cloud processes and cloud feedbacks in large scale models, Reading, 1999. 
Murray, J. D.: Mathematical Biology, Springer, Berlin, 1993.

Naveau, P. and Moncrieff, M. W.: A probabilistic description of convective mass fluxes and its relationship to extreme value theory, Q. J. R. Meteorol. Soc., 129, 1-12, 2003

Neggers, R. A. J., Siebesma, A. P., and Jonker, H. H. J.: Size Statistics of Cumulus Cloud Properties in Large-Eddy Simulations, J. Atmos. Sciences, 60, 1060-1074, 2002.

Ogura, Y. and Takahashi, T.: Numerical simulation of the life cycle of a thunderstorm cell, Mon. Wea. Rev., 99, 895-911, 1971.

Haken, H.: Synergetics, An Introduction, Springer, Berlin, Heidelberg, New York, 1977.

Haken, H.: Advanced Synergetics, Springer, Berlin, Heidelberg, New York, 1983.

Nordeng, T.: Extended versions of the convective parameterisation scheme at ECMWF and their impact on the mean and transient activity of the model in the tropics, Tech Memo 206, 41 pp., Europ. Centre Medium Range Weather Forecasts, Reading, England. 1994

Roeckner, E., Arpe, K., Christoph, M., et al.: The atmospheric general circulation model ECHAM4: Model description and simulation of present day climate, Max-Planck-Institut Report, 218, 90 pp., 1996.
Siebesma, A. P. and Holstlag, A. A. M.: Model impacts of entraining and detraining rates in shallow cumulus convection, J. Atmos. Sci., 53, 2354-2364, 1996.

Tiedtke, M.: A comprehensive mass flux scheme for cumulus cloud parameterisation in lagre-scale models, Q. J. R. Meteor. Soc., 117, 1779-1800, 1989.

Tompkins, A.: A prognostic parameterisation for the subgrid-scale variability of water vapor and clouds in large scale models and its use to diagnose cloud cover, J. Atmos. Sci., 59, 1917-1942, 2002.

Volterra, V.: Lecons sur la Theorie Mathematique de la Lutte pour la Vie, Gauthier Villars, Paris, 1931.

Weinstein, A. I. and MacCready, P. B.: An isolated cumulus cloud modification project, J. Appl. Met., 8, 936-947, 1969.

Weger, R. C., Lee, J., Zhu, T., and Welch, R. M.: Clustering, Randomness and Regularity in Cloud Fields: 1. Theoretical Considerations, J. Geophys. Res., 97, 20 519-20 536, 1992.

Weger, R. C., Lee, J., and Welch, R. M.: Clustering, Randomness and Regularity in Cloud Fields: 3. The Nature and Distributions of Clusters, J. Geophys. Res., 98, 18 449-18 463, 1993.

Zhang, G. J.: Convective quasi-equilibrium in midlatitude continental environment and its effect on convective parameterization, J. Geophys. Res., 107(D14), doi:10.1029/2001JD001005, 2002. 